\title{
Agricultural Stakeholders' Perceptions of Occupational Health and Safety in the Southeastern US Coastal States ${ }^{1}$
}

\author{
Tracy Irani, Beatrice Fenelon Pierre, and Tyler S. Nesbit ${ }^{2}$
}

\section{Introduction}

Agriculture is one of the most dangerous industries globally for worker health and safety. Risks to occupational health and safety include adverse weather, intense labor, and exposure to mechanical, biological, and chemical dangers. US agricultural workers suffer the highest rate of heat-related fatalities of any industry, with a rate of 3.06 deaths per million workers per year (Gubernot et al., 2015). Symptoms ranging from headache and dizziness to dehydration and heat stroke are common examples of heat-related illness (HRI) associated with exposure to extreme heat and intense working conditions (Fleischer et al., 2013). Furthermore, recent research has highlighted the challenges to mental health that agricultural workers face due to a variety of occupation-related stressors (Cole \& Bondy, 2020). In addition to the inherent dangers of modern production agriculture, the prevalent socioeconomic conditions of agricultural workers also impact occupational health and safety. For example, insecure housing arrangements, such as tenuous rental agreements and overcrowding, constitute an additional stressor on workers and their families. Deficient housing conditions, such as lack of access to hot and cold water and handwashing sinks, also pose a direct threat to health and safety by limiting proper sanitation and pesticide safety practices (Arcury et al., 2017; Levesque et al., 2012).
Despite efforts to address the high levels of occupationrelated illness and injury through regulatory and educational efforts as well as training programs, these issues continue to exact an elevated cost to human health and economic productivity worldwide. These outcomes have been worsened by the COVID-19 pandemic, because many of the Centers for Disease Control and Prevention (CDC) guidelines have been difficult to implement among agricultural workers (Irani et al., 2020).

Therefore, the present study aimed to answer the following four questions:

1. How do farm stakeholders located in the southeastern United States perceive the agricultural industry from an occupational health and safety standpoint?

2. How do they think their industry is trying to improve occupational health and safety?

3. What are the main barriers to implementing occupational health and safety in their industry?

4. What measures do they think could be taken to ensure better occupational health and safety for people working in their industry?

1. This document is FCS3361, one of a series of the Department of Family, Youth and Community Sciences, UF/IFAS Extension. Original publication date October 2021. Visit the EDIS website at https://edis.ifas.ufl.edu for the currently supported version of this publication.

2. Tracy Irani, professor and chair; Beatrice Fenelon Pierre, doctoral student; and Tyler S. Nesbit, doctoral student, Department of Family, Youth and Community Sciences; UF/IFAS Extension, Gainesville, FL 32611.

The Institute of Food and Agricultural Sciences (IFAS) is an Equal Opportunity Institution authorized to provide research, educational information and other services

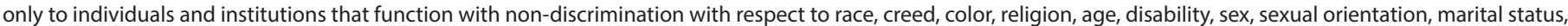

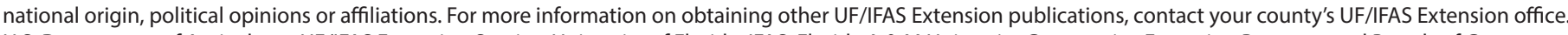
U.S. Department of Agriculture, UF/IFAS Extension Service, University of Florida, IFAS, Florida A \& M University Cooperative Extension Program, and Boards of County Commissioners Cooperating. Nick T. Place, dean for UF/IFAS Extension. 
This article is targeted to agricultural producers, policy makers, and interested residents. We anticipate that our findings will have broader significance in the international context as many challenges to achieving agricultural occupational health and safety extend globally (ILO, 2017).

\section{Methods}

The research team used a qualitative semi-structured interview approach to collect data from 11 participants. The study participants included Extension agents, farmworkers' advocates, growers, farm labor contractors, and representatives from the worker safety program, as well as the forestry and fruit and vegetable associations. They were over 50 years of age and were highly knowledgeable about the farm and forestry industries.

The interview protocol contained eight questions related to occupational health and safety with a special section on COVID-19 because the research was deployed during the pandemic. The researchers conducted the interviews via the online platform Zoom, which both recorded and transcribed the interviews with its built-in features. The data collection process lasted one month (August 2020). For a complete methodology, see Irani et al. (2021).

\section{Results}

This section presents the three main themes that emerged from the participants' responses regarding occupational health and safety within the agricultural industry: 1) the description of the current state of health and safety within the industry, 2) the barriers to promoting occupational health and safety, and 3) the enablers or measures that are underway to offset the identified barriers and to promote health and safety compliance.

\section{Description of the Agricultural Industry}

Three interdependent elements captured the participants' description of the agricultural industry: 1) inherent danger, 2) regulations, and 3) dependence on the agricultural worker population's attitudes and education.

The participants described agriculture as one of the most dangerous industries, not only because of the risky nature of the work, but also because of workers' lack of access to healthcare. The participants reported that agricultural workers operate under hot temperatures for excessively long hours. Sometimes they do not even take their required water break in order to make more money, particularly those who are paid by piece. Also, participants felt workers are sometimes exposed to pesticides that cause multiple diseases, including skin, respiratory, and cardiovascular diseases. One participant reported that: "There are lots of acute effects. Almost every farmworker that works in farm work at some point in their life has rashes that are related to pesticide exposure, of which acute skin diseases and heatrelated illnesses are the two common diseases."

Although farmworkers are exposed to such risks, seldom do they have proper healthcare access. Even those who could be eligible for certain programs often miss such opportunities due to lack of legal documentation and fear of deportation. They are also afraid of using free available services because of the public charge rule, which penalizes ineligible people for using public healthcare services. Here is an excerpt from a participant's response:

"Some workers know where the clinic is, they go to their clinic. Right now, the current administration [as of 2019] and the federal government have caused this thing called the public charge rule. Right now, a lot of farmworkers are afraid to even go to the clinic because they're afraid they're going to be penalized because they're using health care services because of the public charge. So, sometimes people are afraid to go to the clinic because they're afraid of immigration. And sometimes they're afraid to go to the clinic, they don't have health insurance. And sometimes they're afraid to go to the clinic because there has been rumor that the clinics are going to hurt them."

Additionally, the existing agriculture-related regulations do not always seem conducive to occupational health and safety compliance. The participants identified Florida as one of the most challenging states for at-risk agricultural stakeholders, including farmworkers, farmers, and contractors or intermediaries, to maintain standards for occupational health and safety because of the limited intervention of OSHA (Occupational Safety and Health Administration) in the industry. One participant noted that "there is not enough compliance with pesticides regulations. There is not enough enforcement of pesticide regulation." However, they recognize the new Agricultural Worker Protection Standard (WPS), which regulates health and safety, may provide more protection for farmworkers.

Furthermore, the findings suggest that farmworkers' sex, age, motives, agricultural experience, and culture, including their education, attitudes, beliefs, and stigmas, determine their behavior toward health and safety. All the participants perceived men to be less concerned about health and safety than women. However, they all perceived youth, regardless of gender, to be less risk averse than older people, even during the COVID-19 pandemic. Also, new H-2A and newly 
arrived farmworkers were reported to be less cautious and therefore took more risks because of their inexperience and lack of knowledge about the working context. Finally, people from more communal cultures seem to be less likely to comply with health and safety measures, even with the CDC guidelines during the pandemic, particularly with social distancing.

\section{Barriers to Promoting Occupational Health and Safety}

The study participants evoked multiple interconnected and overlapping constraints to implementing occupational health and safety practices in agriculture that we categorized into education, lack of access to healthcare, logistics, discrimination, cultural competency, economic considerations of farm owners and workers, and transparency of labor contracts.

The participants echoed that education is the entry point to implementing health and safety measures. Unfortunately, farmworkers' availability to attend training can be an issue due to cyclical mobility and long hours. Language and literacy limitations as well as inadequate housing conditions, including the lack of reliable Internet access, result in barriers to accessing learning resources and support opportunities. One participant stated: "They come to a seasonal work, and they move to another state, then come back again.... So, that also can affect how you can design a program to ensure that there is a continuing education program that would increase their ... opportunities to improve their life."

Healthcare access constitutes another constraint among both documented and undocumented workers but mostly in the latter category. One participant noted that "they might not be feeling very comfortable to show up in a clinic because they may be undocumented." Even those who have documentation, such as the $\mathrm{H}-2 \mathrm{~A}$ workers, may decline health insurance because of the cost. The labor contracting system that could help establish the party responsible for workers' health and safety was viewed by participants as flawed and even confusing. According to the participants, although the labor contractors are the official employers, sometimes the contractors defer the responsibility to the farm owners and managers. In other cases, the farm owners and managers do the opposite. One participant declared that "Lot of times the grower ... doesn't even know what's going on. Sometimes, they're being pushed by the labor contractor." Another participant explained:
"A large majority of the workforce, particularly in Florida, is managed by farm labor contractors. ... So, it seems to me that is sort of the key to getting to the workers themselves is through the farm labor contractors, the people they work with."

Additionally, participants reported that transportation has a big impact on the workers' healthcare access. One participant revealed that "They don't have transportation, you know. ... somebody has to miss a day of work with them to bring them to a clinic." These circumstances contribute to a lack of preventive care for agricultural workers. "The farmworkers go to the doctor when they are dying. So, they don't do preventive care too much. You know, ... 'Oh, I'm okay. I get a little pain here, and don't want to call until the last minute."

Furthermore, participants mentioned issues related to discrimination and cultural competency that can prevent the industry from engaging in optimal operations. For example, they pointed out racism between farmers and workers, classism among workers, stigma related to cultural norms, and lack of cross-cultural awareness. One participant added, "There is also an issue of classism. We were in one nursery where the supervisors were the same ethnic group. They demeaned the workers under them because they were less educated. There were really big class issues among some of the workers and the supervisors, because the supervisors look down on the workers and call them bad names. There's racist issues, very big problem even between growers and workers, but also among workers sometimes."

Finally, the participants mentioned some economic considerations from both the farmers' and the workers' side that hamper the effectiveness of health and safety efforts. From the workers' side, the findings revealed that farmworkers push themselves to their physical limits-working long hours, skipping water and shade breaks, and working even when sick-to maximize their income. They are responsible not only for themselves, but also for family members living with them as well as those in their home countries. In other words, because of their financial insecurity, workers tend to prioritize work over their health. One participant added, "These guys, you know, if they get a cold, they don't go to the doctor, they want to work, they came here to make money. They make really good money when they're here, certainly, in comparison to what they are able to make where they came from." However, from the farmers' side, the economic tension to implementing health and safety measures stems from the shallow profit margins of agriculture. A participant shared the following regarding the perspective of farm management: "Because this [purchasing personal protective 
equipment] will be seen as impacting their bottom line. We will be seen as costing them time, costing them money."

\section{Enablers to Offset Identified Barriers and to Promote Health and Safety Compliance}

The analysis produced three subthemes of enabling factors for successful integration of occupational health and safety measures into the agricultural industry related to education, regulation, and preventive health care. These include opportunities to improve education through increased and more effective outreach and emphasizing the connection between health and economic opportunity. The role of regulations emerged as an important element in incentivizing and enforcing worker health and safety practices and standards. Improving access to health care, especially preventive care, also emerged as an opportunity to advance occupational health and safety of agriculture workers.

\section{Education}

Findings suggest that education constitutes a potential way to improve health and safety in the agricultural industry. This has been reinforced by the COVID-19 experience. Although COVID-19 has created a lot of disturbance in the industry, as in every business sector, participants agreed that it has provided a new opportunity for training providers and Extension specialists to revamp their programs and adapt their approach. Virtual training sessions were revealed to yield higher efficiency through an increased number of participants. Agricultural stakeholder participants, including farmers, did not question the use of online training. They seemed to be comfortable with such training settings. One participant stated:

"We've moved our longer training program to an online format for this year. And I suspect, and talking to folks, as people get more and more comfortable, that online virtual meeting will become more of a staple even after we go back into a space where we can get together."

As highlighted in the section on barriers, participants mentioned the importance of topics related to racial and ethnic discrimination, classism, and inadequate sanitation, which constituted impediments to proper implementation of occupational health and safety, including the CDC COVID-19 guidelines. Therefore, participants suggested that developing educational materials in these subject areas would help to fully address occupational health and safety in the agricultural industry. Furthermore, participants recommended holding training sessions and providing training material to people in their own languages to ensure optimal knowledge transfer. One participant added:
"We see workers who don't speak English. So, providing training opportunities for them in other languages outside of English would be ideal."

\section{Regulations}

Several participants focused on the role of regulations in improving worker health and safety. This subtheme contained several components, including improving the application and enforcement of existing standards, such as the improved Worker Protection Standard (WPS), Good Agricultural Practices (GAP), and Food Safety Modernization Act (FSMA) regulations, as well as incorporating stronger protections for farmworkers. One participant proposed:

"I think we need policies that protect workers. Workers are being affected by poor health, poor housing, crowded housing, by lack of access to educational material, by heat. We need to come up with a protection plan for workers. Oftentimes I feel like a lot of the laws are designed to protect growers from lawsuits but I think in doing that, we oftentimes also ask again why these protections are in place."

Additionally, broader aspects of regulations were addressed, including a call to acknowledge agricultural workers as a skilled labor force, to standardize a process of on-farm reviews, and to address system-wide issues that inhibit the ability of the industry, as a whole, to provide adequate health and safety measures. This sentiment is captured by the following statement:

"Well, we need to have a different culture [in] our entire agricultural system. ... there's something wrong with our system. If growers are not being able to make enough money ... it's all along the food chain."

\section{Health Care and Prevention}

Healthcare access emerged as a critical element of improving agricultural workers' health and safety. Participants conveyed that addressing agricultural occupational health and safety requires prioritizing farmworkers' healthcare. Some of the opportunities to improve in this area include instituting positions in farm operations to oversee and manage health and safety training and implementation, providing preventive care for uninsured and underinsured workers, and working with healthcare providers to address the specific risks of agricultural work. For many farms, health and safety training may be a one-time event rolled into the overall operation. Respondents shared in interviews that the creation of a health and safety manager 
position may help to bring more focus and consistent implementation of best practices.

"These days you need a position for a safety and health manager and that person will oversee the safety and health program for the company. When you see [a] company [without] that person in place you will see more problems, you know."

One of the responsibilitiesof a health and safety manager is to provide an increased focus on preventive care for workers, which would reduce the need for emergency services. There is an opportunity to expand this focus. One participant shared a positive example of working with not-for-profit volunteer healthcare providers to provide preventive care for their workers:

"We participate with a group of doctors that are better volunteers that come to our job site and test workers and provide dental care once a year, and I think that's very much a positive thing. But if that could be somehow provided to these workers that don't have health insurance, it would be a great benefit to everyone. ... One time a year, we would bring that volunteer group in and they would provide medical services, shots, dental care, and all kinds of things for them."

This example represents a possible way forward in reaching more agricultural workers where they are in terms of addressing their medical needs in their economic reality. Finally, in addition to working with healthcare professionals to provide more accessible services, another opportunity is in working to educate healthcare providers about the specific risks and dangers of agricultural work. For example, the common illnesses associated with pesticide exposure may be misidentified as a common rash. Incorporating knowledge of the industry's practices and associated risks into the training of nurses and doctors, especially those serving in clinics in rural and agricultural areas, would be advantageous to the health of agricultural workers. One participant described providing a training to prepare doctors to treat farmworkers, after which a doctor commented:

"You know, I had a farmworker patient about two weeks ago that had rash all over his body and I just diagnosed it as acute dermatitis, gave him some cream, and sent him home. But after hearing you talk, I think now that was related to pesticide exposure and I should have put it down as pesticide exposure, not acute dermatitis, and I should have reported it and I should have told him to take protections against pesticide."

\section{Conclusion and Recommendations}

Despite the recognition of agriculture as a hazardous industry and efforts to improve occupational health and safety, there is still much work to do. The working conditions can be slow to change despite recent policy efforts to improve worker health and safety. Some of the challenging issues that emerged from our analysis are the slim economic margins within which many farms operate. Access to high-quality, preventive healthcare is also a major concern for farmworkers in the Southeastern coastal states. Finally, barriers related to cultural norms and lack of cross-cultural awareness continue to adversely affect agriculture workers' health and safety. Specific recommendations include the following:

- The best opportunities to improve conditions may be found in innovations to educational practices, including content and materials that encourage an increasing sensitization of farm owners and workers to the importance of health and wellness.

- The critical importance of agricultural worker health and safety has been underscored by the adversities caused by the COVID-19 pandemic, which also highlighted the promise of virtual trainings. Owners, crew leaders, and workers are more accepting of online training than they were pre-pandemic and are more familiar with online tools such as Zoom.

- Additionally, regulatory approaches are needed to provide economic supports to farmers and farmworkers, to eliminate the need to sacrifice health and safety practices for the sake of productivity, and to ensure adequate access to healthcare for agricultural workers, including the large proportion of immigrant farmworkers.

- Based on our analysis, healthcare coverage should be in the forefront of any contractual negotiation between employers and agricultural workers.

\section{Funding}

The authors gratefully acknowledge the support of the Centers for Disease Control and Prevention (CDC)/ National Institute for Occupational Safety and Health (NIOSH) grant 1 - U54 OH 011230 - 01, which funds the Southeastern Coastal Center for Agricultural Health and Safety (http://sccahs.org/). 


\section{Disclaimer}

The contents of this article are solely the responsibility of the authors and do not necessarily represent the official views of the Centers for Disease Control and Prevention or the Department of Health and Human Services.

\section{References}

Arcury, T. A., Trejo, G., Suerken, C. K., Ip, E. H., \& Quandt, S. A. (2017). Stability of Household and Housing Characteristics among 830 Farmworker Families in North Carolina: Implications for Health. Journal of Immigrant and Minority Health, 19 (2), 398-831. https://doi.org/10.1007/ s10903-016-0369-3

Cole, D. C., \& Bondy, M. C. (2020). Meeting Farmers Where They Are-Rural Clinicians' Views on Farmers' Mental Health. Journal of Agromedicine, 25 (1),126-134. https://doi.org/10.1080/1059924X.2019.1659201

Fleischer, N. L., Tiesman, H. M., Sumitani, J., Mize, T., Amarnath, K. K., Bayakly, A. R., \& Murphy, M. W. (2013). Public Health Impact of Heat-Related Illness among Migrant Farmworkers. Am. J. Prev. Med., 44, 199-206.

Gubernot, D. M., Anderson, G. B., \& Hunting, K. L. (2015). Characterizing Occupational Heat-Related Mortality in the United States, 2000-2010: An Analysis Using the Census of Fatal Occupational Injuries Database. Am. J. Ind. Med., 58, 203-211.

International Labor Office. (2017). Working Together to Promote a Safe and Healthy Working Environment. International Labour Conference $106^{\text {th }}$ Session.

Irani, T., Pierre, B. F., \& Nesbit, T. S. (2020). Agricultural Professionals' Perceptions of COVID-19 and Occupational Health and Safety. Journal of International Agricultural and Extension Education, 28 (1) Special Issue. doi: 10.5191/ jiaee.2021.28106.

Irani, T., Pierre, B. F., \& Nesbit, T. S. (2021). Agricultural Stakeholders' Perceptions of Occupational Health and Safety in the Southeastern U.S. Coastal States. International Journal of Environmental Research and Public Health, 18, 6605. https://doi.org/10.3390/ijerph18126605

Levesque, D., Arif, A., \& Shen, J. (2012). Association between Workplace and Housing Conditions and Use of Pesticide Safety Practices and Personal Protective Equipment among North Carolina Farmworkers in 2010.
International Journal of Occupational and Environmental Medicine, 3 (2), 53-67. 